\title{
Design of Dynamic Tangible Workspaces for Games: Application on Robot-Assisted Upper Limb Rehabilitation
}

\author{
Arzu Guneysu Ozgur ${ }^{1}$, Barbara Bruno ${ }^{1,2}$, Victor Taburet ${ }^{1}$, Ayberk Özgür ${ }^{1}$, and Pierre Dillenbourg ${ }^{1}$
}

\begin{abstract}
A key element for the success of any game is its ability to produce a different experience at each round, thus keeping the player engagement high. This is particularly important for those games that also have a serious objective, such as gamified rehabilitation systems, aiming at encouraging patients in performing home rehabilitation exercises. In all cases, a game element which is typically static is the workspace, i.e. the "floor" upon which the game takes place. This is especially true for robot-assisted rehabilitation games, where the workspace must satisfy the requirements given by the robot's locomotion and localization systems, as well as the patient's exercise motion requirements.
\end{abstract}

In this article we present a simple yet effective solution for designing dynamic and customizable tangible workspaces, which relies on hexagonal tiles and our previously proposed Cellulo localization system. These "hextiles" can be easily tangibly rearranged at each game round to yield a desired workspace shape and configuration, allowing tabletop mobile robots to move continuously within each new workspace. We ground our solution in the context of robot-assisted rehabilitation, where high adaptability is crucial for the efficacy of the solution, and propose a dynamic extension of our "tangible Pacman" rehabilitation game.

Experiments show that the proposed solution allows for adaptation in range of motions, exercise types, physical and cognitive difficulty, besides reducing repetitiveness.

\section{INTRODUCTION}

Gamified rehabilitation is the branch of rehabilitation which seeks to increase patients' motivation towards the rehabilitation exercises by introducing game-like elements such as entertaining feedback mechanisms (objectives, rewards and penalties) and automated difficulty adaptation [1], [2]. The motivations supporting gamified rehabilitation are simple and strong: One-to-one rehabilitation sessions for healthcare system are becoming more and more unsustainable, forcing therapists to complement the therapy sessions with home rehabilitation solutions that are to be autonomously and regularly performed by the patient. However, these home exercises are criticized for being frustrating and boring, with the result that approximately only $30 \%$ of the patients carry out the home routines as recommended [3].

Among the characteristics sought by gamified rehabilitation solutions, adaptation deserves special attention: It is a crucial factor for keeping the user engaged with the activity [4], while also being a prerequisite to ensure that the

\footnotetext{
*This work was partially supported by the Swiss National Science Foundation through the National Centre of Competence in Research Robotics.

Authors are with ${ }^{1}$ Computer Human Interaction in Learning and Instruction Laboratory (CHILI), ${ }^{2}$ MOBOTS, Swiss Federal Institute of Technology (EPFL), Lausanne, Switzerland. arzu . guneysu@epfl . ch, [name]. [surname] depfl.ch
}

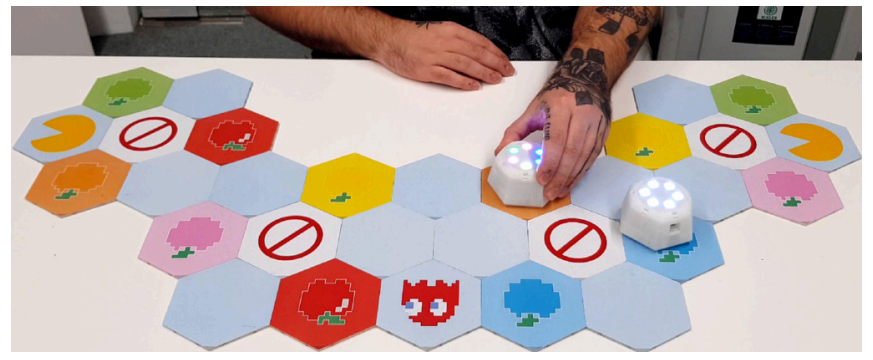

Fig. 1: User playing the "tangible Pacman" game with the Cellulo robots on the dynamic tangible workspace.

activity is tailored to the user's capabilities, characteristics and rehabilitation goals.

Under these premises, robotic rehabilitation solutions are promising thanks to the high number of degrees of freedom they can allow and the wide range of sensors they can embed to detect the user's status and adapt to it [1], [5]-[7].

Specifically considering upper-limb rehabilitation systems, existing solutions can be divided into stationary systems, which typically envision the user to interact with a system acting within a fixed workspace [6], [7], and wearable/portable systems, for which the workspace is defined relative to the user [8]-[10]. An interesting "third way" is the one we proposed with the Cellulo-based tangible Pacman upper limb rehabilitation game [11], which combines the typical setup of stationary solutions (commonly adopted in therapy and therefore familiar to the users) with the ease-of-use of portable systems.

Tangible Pacman can be set up on any flat surface large enough to host one of the game maps (a printed sheet of paper with size $980 \times 420 \mathrm{~mm}$, or $620 \times 420 \mathrm{~mm}$ ) that serves as the workspace for the Cellulo robots. The user has to move a Cellulo robot (the Pacman) in a tangible rendition of the classic Pacman game in which other, autonomous Cellulo robots act as chaser Ghosts [11]-[13]. The map is entirely covered with a micro-dot pattern that ensures sub$\mathrm{mm}$ accuracy in the robots' localization, while the graphical elements can trigger specific behaviours on the robot. As an example, the walls produce a repulsive haptic feedback which guides the user to stay in the corridors, while the fruits, corresponding to target locations to reach, incrementally turn on the LEDs on the top of the robot. Similarly, whenever the chaser catches the user's robot, the user loses all of the fruits collected so far. The interested reader can find more information on the tangible Pacman game in [11] and on the Cellulo robotic platform in [14]. 


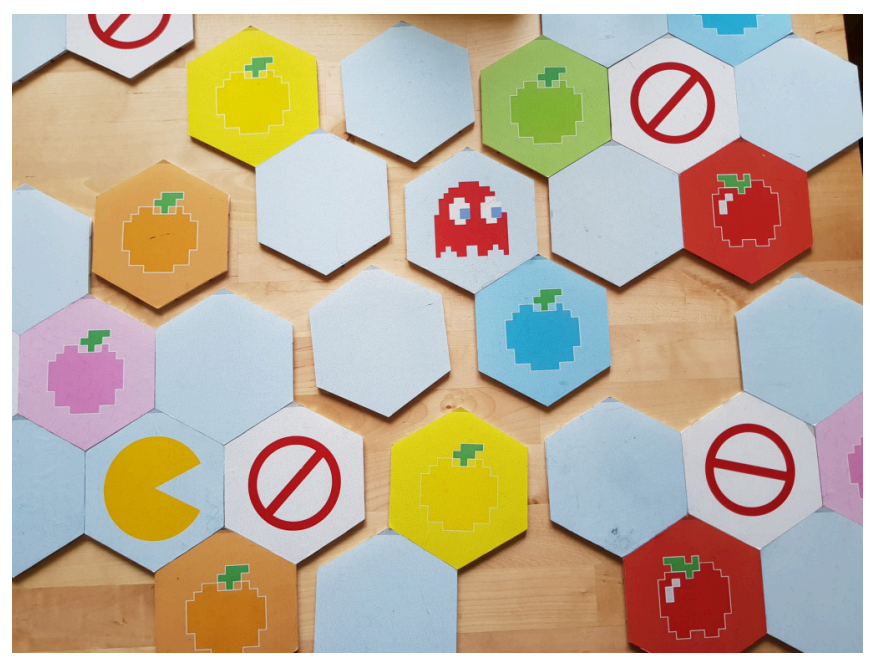

Fig. 2: The hexagonal tiles of the dynamic workspace.

While the game itself allows for adaptation over a number of game elements, such as the number and speed of the ghosts or the robot's haptic feedback to tune the challenge level [11]-[13], it has one crucial element which is not fully adapted: the workspace itself. Since the size of the map is a-priori defined, reaching all of its points (and especially its borders) requires an arm mobility that not all users might possess. Additionally, points of interest (denoted in the map with red fruits) are located in static positions. As experienced during our studies with acute and chronic stroke survivors, these two limitations might reduce the effectiveness of the game towards rehabilitation by preventing a number of users from using it and by possibly inducing boredom due to the repetitiveness in the point of interest locations.

In this article we tackle both limitations discussed above, by proposing a dynamic, customizable workspace made of hexagonal tiles as seen in Fig. 1 and Fig. 2. As the experiments show, the user or therapist can place the tiles in any configuration, which allows for:

- Taking into account the mobility capabilities of the user, e.g. requiring an asymmetric map

- Increasing the engagement of the game, by letting the user place the special tiles in many different ways

- Making the game evolve together with the rehabilitation objectives, e.g. starting with a smaller one and gradually moving towards larger ones, or inducing the user to train specific movements as well as bilateral ones

Although the solution proposed is grounded in the setting of the tangible Pacman rehabilitation game, we see two clear avenues for generalization:

First, within the context of upper-limb rehabilitation, our solution can be ported to any system equipped with a similar localization scheme working on a similar workspace. By allowing the workspace to be tiled and the resulting tiles to be moved around, it could help make the system more engaging and interactive for users.

Second, within the context of robot-enhanced games, the simple use of a dynamic workspace creates another dimen-

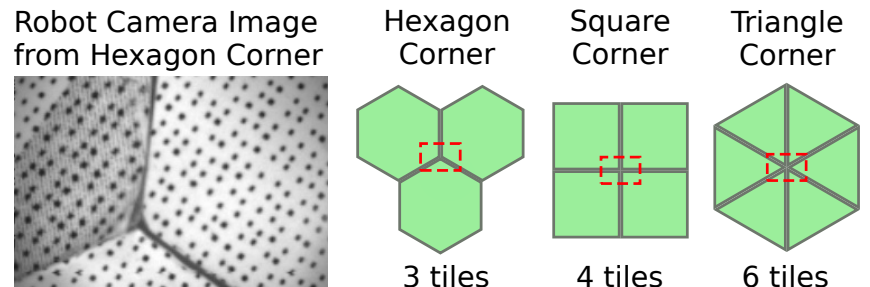

Fig. 3: Robot camera view on a corner with hexagonal tiles. The localization system of the robot requires at least one $8 \times 8$ matrix of adjacent dots, corresponding to a unique $(x, y)$ point, to be visible in the image. The matrix is only valid if all of the points composing it belong to the same continuous space (e.g., the same tile). Each hexagonal tile corner connects with 3 tiles only, which maximizes the continuous visible areas compared to triangle and square corners that have 4 and 6 tiles contacting in each corner respectively.

sion for game design where the building of the workspace is the key creative element. Such a dimension could be employed as e.g a first game phase (which is the creation of the workspace) in addition to the original playing of the game, which becomes the second phase. These configurations could be especially appealing in multiplayer games where players are first cognitively challenged to design a difficult workspace for the opponent, and then to find the best strategy to play on their given workspace.

\section{Design of the Dynamic Tiled Workspace}

\section{A. Theoretical Tessellation Framework}

The Cellulo robots rely on a dotted pattern printed over the entire workspace map for localization [15]. Therefore, the design of a dynamically rearrangeable workspace for Cellulo robots requires a tessellation of the flat game space with basic geometric tangible elements. Tessellation in two dimensional flat surface, also called planar tiling, is a topic in geometry that studies how shapes, known as tiles, can be arranged to fill a plane without any gaps, according to a given set of rules. Common rules are that there must be no gaps between tiles, and that no corner of one tile can lie along the edge of another [16]. Furthermore, a regular tessellation with identical regular polygons is desirable when building the workspace, as less complex shapes are well known to decrease cognitive load [17]. Such a tesellation would have both identical regular tiles and identical regular vertices, having the same angle between adjacent edges for every tile [18]. There are only three shapes that can form such regular tessellations: equilateral triangles, squares, and regular hexagons. Any one of these three shapes can be infinitely replicated to fill a plane with no gaps [19].

The Cellulo localization performance correlates with the integrity of the pattern: in the best case it allows for continuous sub-millimetric accuracy [15]. However, the tessellation of the workspace directly implies discontinuities due to the dynamic rearrangement of the tiles. Among the three shapes 


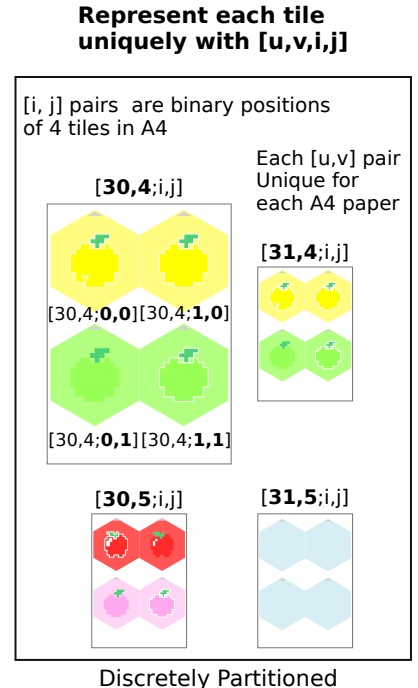

Source Space $[u, v, i, j]$
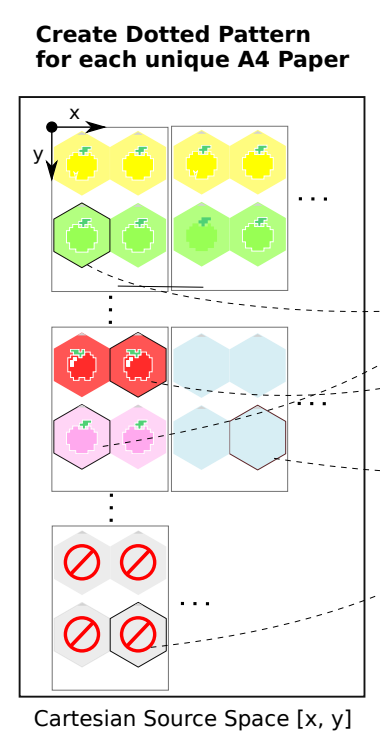

Print Dotted A4 Design on Sticky Papers, 3D print hextiles, Build a Game Map with Hextiles

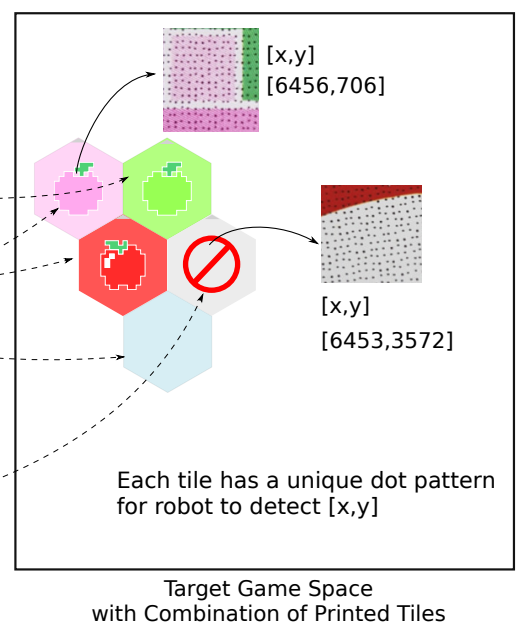

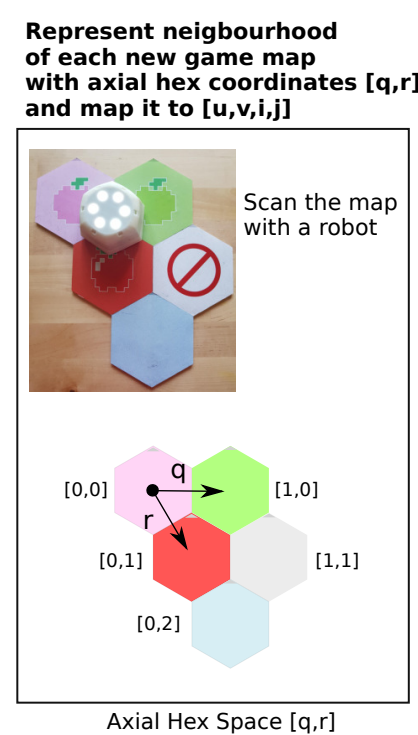

Fig. 4: Representation of tiles in source and target spaces. The cartesian source space is partitioned into A4 sheets with coordinates $[u, v]$ where each sheet fits 4 tiles with coordinates $[i, j]=[0,0] ;[0,1] ;[1,0] ;[1,1]$. This gives each tile a unique set of source coordinates $[u, v, i, j]$ and a well defined way to print their graphical elements together with the Cellulo localization pattern corresponding to the millimetric cartesian source space $[x, y]_{\text {source }}, 4$ tiles at a time. Tiles are arbitrarily arranged by the user in the target space containing its own millimetric cartesian space and its own discrete axial coordinates $[q, r]$, according to the desired source-to-target mapping (i.e $[u, v, i, j]$ to $[q, r]$ ). The robot, moving on the tiles in the target space, scans the dots and obtains the raw millimetric coordinates $[x, y]_{\text {source }}$. Since each such coordinate belongs to a unique tile by design, the scanned coordinates are converted to the target cartesian coordinates $[x, y]_{\text {target }}$ using the $[u, v, i, j]$ to $[q, r]$ mapping. This results in a seamless cartesian target space where the mapping is done transparently so that the robots can move across tiles continuously and report their target coordinates in millimeters.

mentioned above, the hexagon was selected as the tile shape to minimize these discontinuities in space. This issue is most pronounced on the corners, where hexagon tiles provide the least disturbance to the pattern (see Fig. 3).

The hexagonal tiles, henceforth referred to as "hextiles", contain the localization pattern belonging to the initial source space on top of any desired graphical element (see Fig. 2). As they are arbitrarily rearranged to yield a desired workspace, they generate a new target space. It is desirable to have also in this new space a continuous cartesian coordinate system to allow for regular robot motion algorithms and continuous pose data processing. For this, each tile must cover a unique source coordinate area that does not overlap with any other tile. Knowing the exact rearrangement from the source space to the target space, the unique non-overlapping source coordinates obtained from the robot can be directly translated to the continuous target coordinates.

\section{B. Practical Implementation}

The practical implementation is summarized in Fig. 4. The first step involves printing the localization pattern on paper sheets. To maximize the manufacturability of these tiles using easy to access materials, the unique partitioning of the source space was centered around A4 sheets, which fit 4 hextiles with reasonable size. These dotted sheets are generated as PDFs by a script that takes the desired $[u, v]$ coordinates set to uniquely identify that sheet as input, and then printed on self-adhesive A4 sheets. The hexagonal areas on the sheets are then cut and glued onto appropriately sized tangible hextiles (e.g., 3D-printed).

A convenient, fast and robust attachment of tiles to each other is necessary to maximize usability when building a workspace and using it with real robots. To this end we propose symmetric magnetic attachment points on tile edges. Magnetism was preferred over mechanical attachment structures to keep the tile geometry simple and to allow for removal of fully surrounded tiles from their place without compromising the regular hexagonal shape of the top face, which monolithic mechanical structures would not allow. The magnet strength (correlated with its size) was adjusted to balance the attachment strength between easy removal and stability during use. The overall scheme is shown in Fig. 5.

Before an activity, the user physically builds a desired target workspace with hextiles, whose configuration is unknown to the application controlling the robots. This mapping must therefore be decoded and loaded into the application at runtime. Here, autonomous robot exploration cannot be used to map the workspace as the workspace shape is unknown at runtime; an autonomous robot risks falling off of the edge of the workspace before reaching the hypothetical next tile.

To this end, we propose an "autobuilding" phase where the user manually scans the target workspace with a Cellulo robot before the activity. Specifically, after laying down the tiles, the user sets the controller application in autobuild 


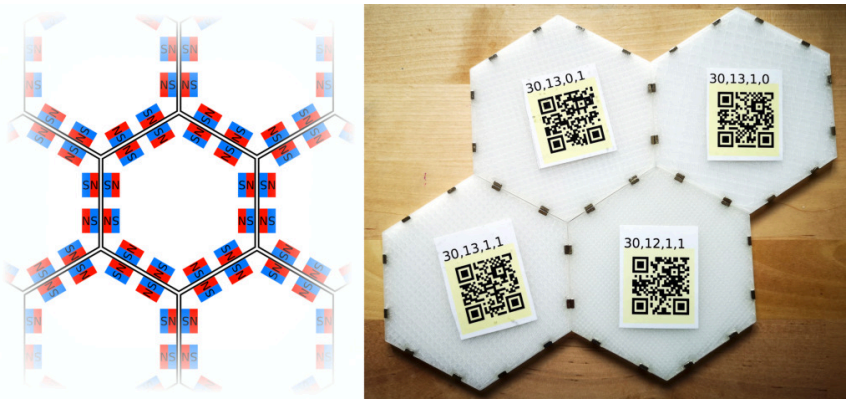

Fig. 5: Tile attachment design (left). Each tile edge contains two magnets close to the exterior, whose pole axes are aligned orthogonal to the edge. On an edge, upper and lower magnets have their north and south poles (or vice versa, for the entire collection of tiles) respectively facing the outside. Each tile thus contains 12 magnets, with 6-way radial symmetry which allows adjacent tiles to attract and remain attached until pulled apart. Real implementation with neodymium block magnets glued into slots in 3D-printed tiles, bottom face up (right). Also seen are its $[u, v, i, j]$ coordinates, for quick identification of many tiles by humans. This setup allows the user to combine tiles comfortably and quickly, as the tiles self-correct their alignment magnetically.

mode, manually places a robot on any tile and moves it continuously from one tile to the next. Among the 6 possible relative placements of each new tile with respect to the previously visited one, the one that minimizes the distance between the last received robot pose on the previous tile and the first received robot pose on the current tile is selected. The mapping is built progressively until all tiles are scanned.

This method is robust as long as the robot is not moved unreasonably fast in order to allow for obtaining pose samples that are close to the edges joining the scanned tiles. Apart from this, other scanning methods may be used to build the mapping, such as a second larger pattern overlaid onto the tiles that is decoded directly by the application through a camera, in one step. Finally, the mapping may be entered manually by the user into the application, or stored in a digital file and loaded if desired.

\section{Tangible Pacman Game with Dynamic Maps}

\section{A. Game Design}

The dynamic workspace designed for the new version of the tangible Pacman game is composed of 10 different types of hextiles, shown in Fig. 2. These are:

- Path hextile is the most basic tile in the game, both the user's robot and the chasing ghost robots can traverse it freely.

- Pacman hextile indicates where users have to position their Cellulo robot at the beginning of each game, it acts as a path tile during the game (which is why it features the same light blue background of path tiles).

- Ghost hextile indicates where users have to position the chasing ghost robot at the beginning of each game, it also acts as a standard path tile during the game.
- 6 different Target fruit hextiles denote locations that the user's robot has to reach during the game. The rationale for having multiple types of targets (denoted by different colors) is again to increase the adaptability and variability of the game. Some of the ways this could be used for adaptation is to have the user collect some subset selectively, use both hands with two robots to collect two fruits simultaneously. These optional adaptations could be correlated with cognitive and/or physical impairments (such as in stroke rehabilitation), as well as with bilateral coordination, respectively.

- Forbidden hextiles play the role of the "walls" in the original tangible Pacman game. Neither ghosts nor the user's robot are allowed to move into such tiles. If the user enters a forbidden tile, they will be penalized by losing a point/fruit and haptic assistive feedback will be activated on their robot to push it out of the tile.

\section{B. Game Rules}

The user's robot, "Pacman", is manually moved by the user on the workspace. One or more distinct Cellulo robots, "ghosts", are controlled by the controller application to move around the workspace aiming at getting close enough to the Pacman to "steal its fruits". The speed of the ghosts can be modified in the controller application to adjust the difficulty of the game. Once the game starts (with all robots in their respective starting positions), all robots are required to always stay flat (camera facing downwards) on the workspace: it is not allowed for the user to "cheat" by lifting any of the robots while the game is on.

Two game elements deserve particular attention:

1) Ghost Behaviour - The current ghost behavior relies on the shortest path algorithm used in the original tangible Pacman game. Ghost chases the Pacman along the path tiles and when it is closer than a pre-defined threshold to the Pacman, it "steals" a pre-defined number of fruits from those collected by the user, who therefore will have to collect again. Both the threshold and the number of fruits stolen are adjustable in the controller application, to allow for difficulty adaptation. Upon catching the Pacman, the ghost automatically turns back to its starting position and, once reached, reprises its chase.

2) Haptic Informative Feedback for Forbidden Hextiles In addition to losing a customizable number of fruits, whenever the Pacman is moved by the user on a forbidden hextile, a haptic feedback is activated on the robot pushing it toward the previous visited tile (i.e. in the opposite direction with respect to the user's movement).

Besides the above general rules, specific variants concerning the number of Pacmans (and ghosts) in the game and the way the user collects fruits can be defined in specific game modes. So far we have implemented two game modes:

1) Single Pacman Mode. This single-player, single-hand mode, containing only one Pacman and one or more 

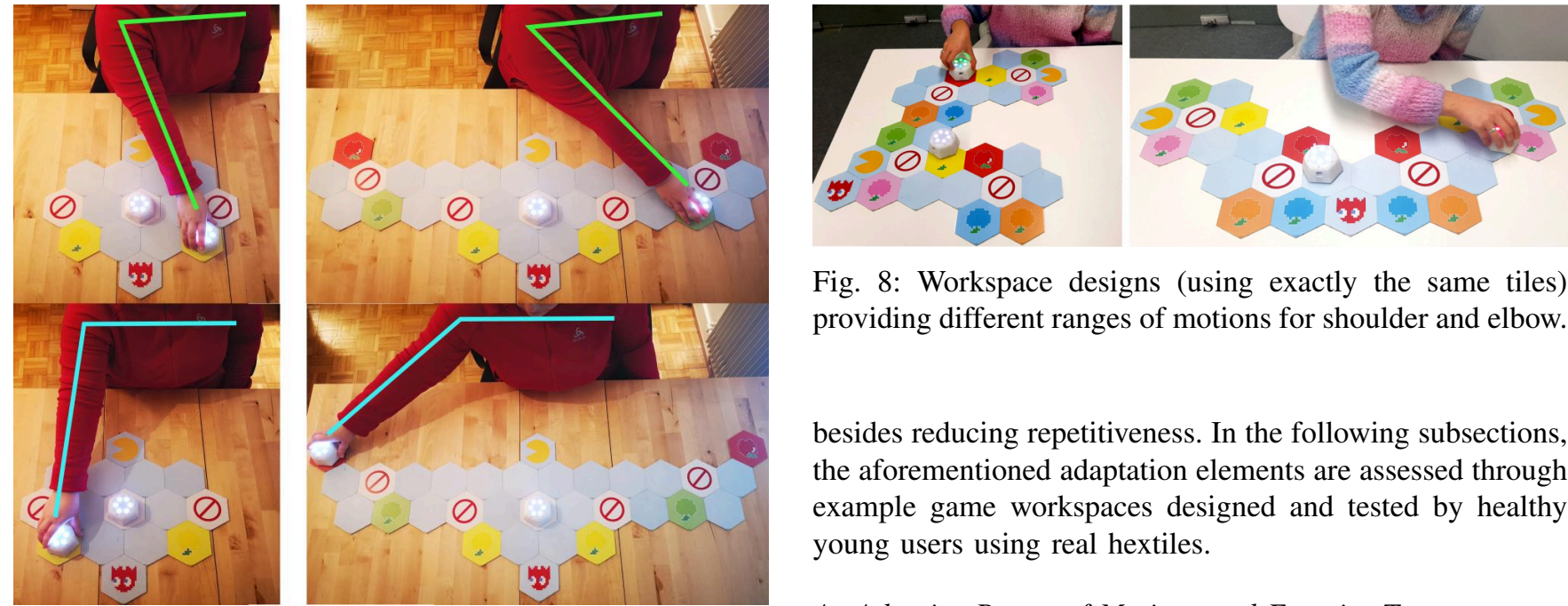

Fig. 8: Workspace designs (using exactly the same tiles) providing different ranges of motions for shoulder and elbow.

Fig. 6: Two workspace designs with different horizontal lengths to provide different ranges of motion for shoulder horizontal adduction (bringing the arm towards the middle of the body around the chest level, denoted with green lines in the top figures) and abduction (the opposite movement, denoted with cyan lines in the bottom figures).

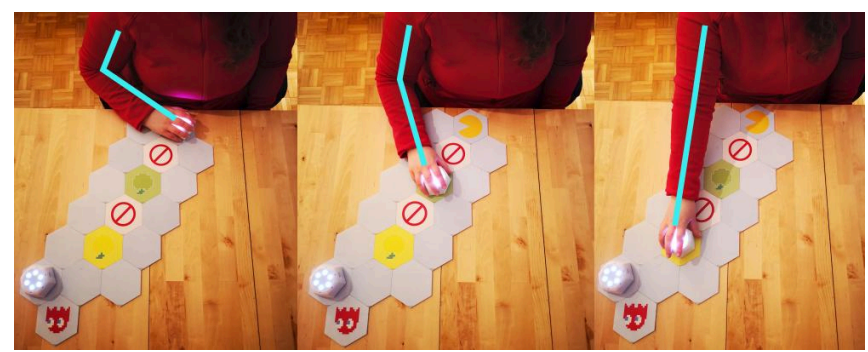

Fig. 7: A workspace designed to specifically train elbow extension (e.g. the straightening of the arm at the elbow, the extension angle denoted with cyan lines).

ghosts, is the simplest version of the game. The user collects fruits by reaching their tile with the Pacman and wins as soon as all fruits in the workspace have been collected. Each collected fruit appears as a bright LED on the top of the Pacman robot, coloured with the color of the collected fruit.

2) Bilateral Exercise Mode. This mode envisions two Pacmans to be controlled by the both hands of the user and, again, one or more ghosts chasing them. In this mode, the user collects fruits only if the two Pacman robots are located on target tiles of the same color, at the same time. This makes the collection harder, as the user is now expected to coordinate the movements of the two arms. Ghosts follow the closest Pacman.

\section{Functional Evaluation}

The rationale for having a dynamic workspace that can be shaped in various ways is that it increases the adaptability of the rehabilitation game, especially in terms of range of motion, exercise type, physical and cognitive difficulty,

besides reducing repetitiveness. In the following subsections, the aforementioned adaptation elements are assessed through example game workspaces designed and tested by healthy young users using real hextiles.

\section{A. Adaptive Range of Motions and Exercise Type}

In the context of rehabilitation, tuning the shape of the workspace allows for tuning the type of movements that will be performed by the patient, specifically adapting to the capabilities and progression of the patient. In our case, modifying the game space directly influences the range of motions that the patient will work with and the intensity of movements necessary to finish the game. This adaptivity is simply provided by the positioning of the target tiles, at different reaching distances both vertically and horizontally. For example:

- Increasing the horizontal size of the workspace and placing the target tiles at the horizontal extremes increases the shoulder horizontal adduction and abduction angles required to reach the left and right extremes. An example comparison is shown in Fig. 6.

- Increasing the vertical size of the workspace and placing the target tiles at the furthest points allows for more elbow extension. Conversely, positioning the target hextiles closer to the torso of the user allows for more elbow flexion (see Fig. 7 and Fig. 8).

- Asymmetric workspaces, with more tiles and targets closer to the affected arm, can be used with people having a reduced range of motion.

- In the Bilateral Exercise Game Mode, the workspace can be asymetrically designed, to suit the motion ranges and rehabilitation goals of the two arms. Playing the map in bilateral mode also enables to increase coordination of the hands. As shown in Fig. 9, this mode also enables a multiplayer version of the game, where the two Pacman robots are controlled by two different users. The workspace can thus be adapted to their different arm lengths, ranges of motions and rehabilitation objectives.

\section{B. Adaptive Difficulty}

While adaptation in terms of physical difficulty is inherently addressed by the adaptation to multiple ranges and types of motions, discussed above, the number and the frequency of unique tiles in the game can provide cognitive 


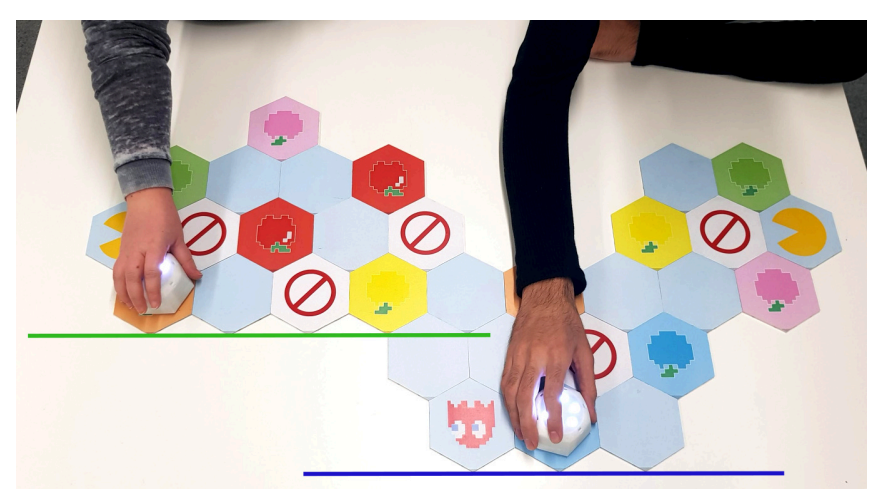

Fig. 9: The dynamic workspace adapted for two players with different arm lengths sitting side by side during a mutliplayer variant of the Bilateral Exercise Game Mode. The green line denotes the range of reach motion for the user on the left, while the blue line denotes the range of motion for the user on the right, having a longer arm.

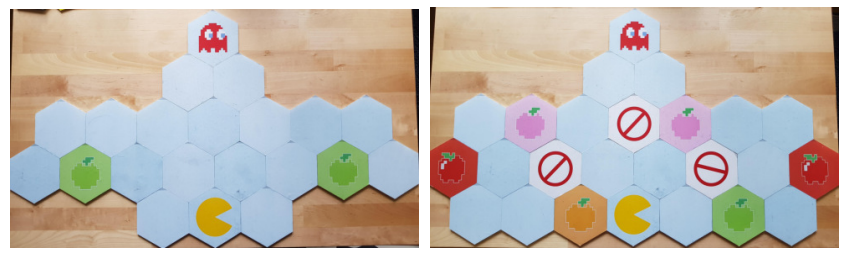

Fig. 10: Workspace designs with different cognitive difficulty. Fewer targets and fewer or no forbidden tiles provide a cognitively easier version of the game.

adaptation. We postulate that the following design choices might enable adaptive cognitive difficulty:

- The number of target fruit tiles having the same colors

- The number of target fruits having different colors

- The presence of forbidden tiles and the corresponding avoidance rule in the game

- The number of forbidden tiles in the game

- Whether the game requires the use of one or both hands, that trains coordination

- The speed of the ghost robot, that trains divided attention, requiring to concurrently keep track of the location of the targets and the ghost, and plan a path towards the chosen target which avoids the ghost

Two example workspace designs with similar physical difficulty and different cognitive difficulty, determined by the items listed above, are shown in Fig. 10.

\section{Decreasing Repetitiveness}

The possibility to freely change the workspace of the game prevents the game from getting repetitive after some rounds. With each new game space, the user needs to adapt to its organisation and new physical and cognitive challenges.

\section{USABILITy EVALUATION}

To validate the user interactions with the developed dynamic tangible Pacman game, we conducted a preliminary usability study with four healthy young users.
During the study, the participants were asked to play the Single Pacman Game Mode, each time freely designing the game workspace using all or a subset of the provided deck of hextiles, or keeping the previous workspace. In each run we recorded the total number of unique tiles visited, the total number of movements from one tile to another (total number of crossings from one tile to another), as well as the farthest tile reached. The data corresponding to the 12 game spaces designed by the participants are shown in Table I.

As the Table shows, the number of unique tiles visited, the number of movements between tiles as well as the farthest tile reached considerably vary across the runs as well as across the map types. Increasing the total number of hextiles used in a workspace increases the number of the farthest tile reached. However, more importantly the number of the farthest tile reached ranges from 8 to 12 within four different workspace designs, even though they use the same number of hextiles (29). This shows that the combination and vertical/horizontal positioning of the hextiles can vary the range of reaching motion. The total number of movements performed by the users goes from 9 to 98 , with the highest numbers occurring in the runs in which the users were caught by a ghost. Since they had to go back and recollect the lost targets, their total motion were increased. Lastly, as expected, the total number of targets affects both the total motion and the total number of unique tiles visited.

These results are preliminary; a more comprehensive analysis of the relationship between design elements and the output metrics should be performed in a controlled study, with a much larger participant population size. However, these data show how the range of reaching motions, as well as the total number of motions, can vary depending on the workspace configuration. This supports the argument made in Section IV.

\section{CONCLUSION}

In this article we proposed the use of tile-based, dynamic and customizable workspaces to increase the adaptability of upper-limb gamified rehabilitation systems, both from a physical and cognitive perspective.

While our work is grounded in the specific robotic platform of the tangible Pacman game, leading to the design of the new dynamic tangible Pacman game, the procedure we outline and its advantages can be ported to (i) any stationary upper-limb rehabilitation system, where a camera can be moved over the workspace to compute the mapping between the fixed source space and the chosen target space; (ii) any game designed for the Cellulo robotic platform, or even for other robots and devices exploiting the same localization principle.

Indeed, the many opportunities for workspace design can be used to create a new generation of interactive, tangible games in which the initial setup of the game elements is an engaging and challenging game in itself.

The reported experimental evaluation hints at the feasibility of the solution we propose and its compliance with the motivating objectives of this work: to allow for adaptation 


\begin{tabular}{|c|c|c|c|c|c|c|c|c|c|}
\hline User Id & Run Id & Map Id & Rows x Columns & \# Hextiles & \# Target & $\begin{array}{r}\begin{array}{r}\text { Unique tiles } \\
\text { visited }\end{array} \\
\end{array}$ & Total tile crossings & $\begin{array}{r}\text { Farthest tile } \\
\text { reached }\end{array}$ & Caught \\
\hline S01 & 1 & $\mathrm{a}$ & $7 \times 12$ & 36 & 12 & 24 & 35 & 14 & No \\
\hline S01 & 2 & $\mathrm{a}$ & $7 \times 12$ & 36 & 12 & 29 & 55 & 14 & Yes \\
\hline S01 & 3 & $\mathrm{~b}$ & $5 \times 10$ & 29 & 12 & 24 & 98 & 12 & Yes \\
\hline S01 & 4 & b & $5 \times 10$ & 29 & 12 & 22 & 30 & 12 & No \\
\hline S01 & 5 & $\mathrm{c}$ & $4 \times 11$ & 30 & 12 & 29 & 66 & 13 & No \\
\hline S01 & 6 & d & $8 \times 9$ & 29 & 12 & 23 & 38 & 10 & No \\
\hline S01 & 7 & $\mathrm{e}$ & $10 \times 7$ & 29 & 12 & 22 & 40 & 9 & No \\
\hline S01 & 8 & f & $8 \times 9$ & 22 & 9 & 17 & 21 & 9 & No \\
\hline S02 & 1 & $\mathrm{~g}$ & $8 \times 6$ & 29 & 6 & 14 & 17 & 8 & No \\
\hline S02 & 2 & $\mathrm{~h}$ & $4 \times 4$ & 12 & 4 & 8 & 9 & 5 & No \\
\hline S02 & 3 & $\mathrm{i}$ & $7 \times 5$ & 19 & 6 & 16 & 22 & 7 & No \\
\hline $\mathrm{S} 02$ & 4 & $\mathrm{j}$ & $7 \times 5$ & 18 & 6 & 14 & 14 & 7 & No \\
\hline S02 & 5 & $\mathrm{k}$ & $7 \times 6$ & 27 & 6 & 17 & 23 & 6 & No \\
\hline S02 & 6 & $\mathrm{k}$ & $7 \times 6$ & 27 & 6 & 23 & 59 & 7 & Yes \\
\hline S02 & 7 & f & $8 \times 9$ & 22 & 9 & 16 & 18 & 9 & No \\
\hline S02 & 8 & $\mathrm{~b}$ & $5 \times 10$ & 29 & 12 & 25 & 91 & 12 & Yes \\
\hline S02 & 9 & b & $5 \times 10$ & 29 & 12 & 22 & 29 & 12 & No \\
\hline S02 & 10 & c & $4 \times 11$ & 30 & 12 & 20 & 26 & 13 & No \\
\hline $\mathrm{S} 03$ & 1 & 1 & $5 \times 10$ & 30 & 12 & 23 & 26 & 11 & No \\
\hline S04 & 1 & $\mathrm{k}$ & $7 \times 6$ & 27 & 6 & 18 & 24 & 6 & No \\
\hline S04 & 2 & $\mathrm{k}$ & $7 \times 6$ & 27 & 6 & 20 & 40 & 6 & Yes \\
\hline S04 & 3 & $\mathrm{k}$ & $7 \times 6$ & 27 & 6 & 18 & 29 & 6 & No \\
\hline S04 & 4 & f & $8 \times 9$ & 22 & 9 & 16 & 21 & 9 & No \\
\hline S04 & 5 & $\mathrm{~b}$ & $5 \times 10$ & 29 & 12 & 25 & 55 & 12 & Yes \\
\hline S04 & 6 & $\mathrm{c}$ & $4 \times 11$ & 30 & 12 & 20 & 26 & 13 & No \\
\hline
\end{tabular}

TABLE I: Data retrieved from the usability study showing the results using the Single Pacman Game Mode with different workspaces designed by the participants themselves, as well as with previously designed workspaces by other participants.

in the range of motions, motion exercise type and cognitive difficulty. Additionally, the variety of game spaces that can be tried by the users can turn each game round into a unique experience, a factor that we believe could positively affect their engagement with and commitment to the rehabilitation exercise and is of crucial importance towards the effectiveness of home rehabilitation solutions.

\section{REFERENCES}

[1] P. Tamayo-Serrano, S. Garbaya, and P. Blazevic, "Gamified in-home rehabilitation for stroke survivors: analytical review," International Journal of Serious Games, vol. 5, no. 1, pp. 2384-8766, 2018.

[2] C. A. Aguilar-Lazcano, E. J. Rechy-Ramirez, H. Hu, H. V. RiosFigueroa, and A. Marin-Hernandez, "Interaction modalities used on serious games for upper limb rehabilitation: a systematic review," Games for health journal, vol. 8, no. 5, pp. 313-325, 2019.

[3] B. Bonnechere, B. Jansen, L. Omelina, and S. V. S. Jan, "Do patients perform their exercises at home and why (not)? a survey on patient habits during rehabilitation exercises," The Ulutas Medical Journal, vol. 2, no. 1, pp. 41-46, 2016.

[4] E. Flores, G. Tobon, E. Cavallaro, F. I. Cavallaro, J. C. Perry, and T. Keller, "Improving patient motivation in game development for motor deficit rehabilitation," in Proceedings of the 2008 international conference on advances in computer entertainment technology, pp. 381-384, 2008.

[5] P. Maciejasz, J. Eschweiler, K. Gerlach-Hahn, A. Jansen-Troy, and S. Leonhardt, "A survey on robotic devices for upper limb rehabilitation," Journal of neuroengineering and rehabilitation, vol. 11, no. 1, p. 3, 2014.

[6] O. Mubin, F. Alnajjar, N. Jishtu, B. Alsinglawi, and A. Al Mahmud, "Exoskeletons with virtual reality, augmented reality, and gamification for stroke patients' rehabilitation: Systematic review," JMIR rehabilitation and assistive technologies, vol. 6, no. 2, p. e12010, 2019.

[7] R. Gassert and V. Dietz, "Rehabilitation robots for the treatment of sensorimotor deficits: a neurophysiological perspective," Journal of neuroengineering and rehabilitation, vol. 15, no. 1, p. 46, 2018.

[8] A. Guneysu, B. Arnrich, and C. Ersoy, "Children's rehabilitation with humanoid robots and wearable inertial measurement units," in 2015 9th International Conference on Pervasive Computing Technologies for Healthcare (PervasiveHealth), pp. 249-252, IEEE, 2015.
[9] M. Kytö, L. Maye, and D. McGookin, "Using both hands: tangibles for stroke rehabilitation in the home," in Proceedings of the 2019 CHI Conference on Human Factors in Computing Systems, pp. 1-14, 2019.

[10] H. Tannous, D. Istrate, A. Perrochon, J.-C. Daviet, A. Benlarbi-Delai, J. Sarrazin, M.-C. H. B. Tho, and T. T. Dao, "Gamerehab@ home: a new engineering system using serious game and multi-sensor fusion for functional rehabilitation at home," IEEE Transactions on Games, 2019.

[11] A. Guneysu Ozgur, M. J. Wessel, W. Johal, K. Sharma, A. Özgür, P. Vuadens, F. Mondada, F. C. Hummel, and P. Dillenbourg, "Iterative design of an upper limb rehabilitation game with tangible robots," in Proceedings of the 2018 ACM/IEEE International Conference on Human-Robot Interaction, pp. 241-250, ACM, 2018.

[12] A. Guneysu Ozgur, L. P. Faucon, P. Maceira-Elvira, M. J. Wessel, W. Johal, A. Özgür, A. Cadic-Melchior, F. C. Hummel, and P. Dillenbourg, "Towards an adaptive upper limb rehabilitation game with tangible robots," in 2019 IEEE 16th International Conference on Rehabilitation Robotics (ICORR), pp. 294-299, IEEE, 2019.

[13] A. Guneysu Ozgur, M. J. Wessel, T. Asselborn, J. K. Olsen, W. Johal, A. Özgür, F. C. Hummel, and P. Dillenbourg, "Designing configurable arm rehabilitation games: How do different game elements affect user motion trajectories?", in 201941 st Annual International Conference of the IEEE Engineering in Medicine and Biology Society (EMBC), pp. 5326-5330, IEEE, 2019.

[14] A. Özgür, S. Lemaignan, W. Johal, M. Beltran, M. Briod, L. Pereyre, F. Mondada, and P. Dillenbourg, "Cellulo: Versatile handheld robots for education," in Proceedings of the 2017 ACM/IEEE International Conference on Human-Robot Interaction, pp. 119-127, ACM, 2017.

[15] L. Hostettler, A. Özgür, S. Lemaignan, P. Dillenbourg, and F. Mondada, "Real-time high-accuracy $2 \mathrm{~d}$ localization with structured patterns," in Robotics and Automation (ICRA), 2016 IEEE International Conference on, pp. 4536-4543, IEEE, 2016.

[16] J. H. Conway, H. Burgiel, and C. Goodman-Strauss, The symmetries of things. CRC Press, 2016.

[17] R. Stavy and R. Babai, "Complexity of shapes and quantitative reasoning in geometry," Mind, Brain, and Education, vol. 2, no. 4, pp. 170-176, 2008.

[18] H. S. M. Coxeter, Regular polytopes. Courier Corporation, 1973.

[19] J. Gullberg, Mathematics: from the birth of numbers. WW Norton \& Company, 1997. 